Dynamics of inertial particles in free jets

This article has been downloaded from IOPscience. Please scroll down to see the full text article.

2010 Phys. Scr. 2010014001

(http://iopscience.iop.org/1402-4896/2010/T142/014001)

View the table of contents for this issue, or go to the journal homepage for more

Download details:

IP Address: 151.100.121.159

The article was downloaded on 07/01/2011 at 16:00

Please note that terms and conditions apply. 


\title{
Dynamics of inertial particles in free jets
}

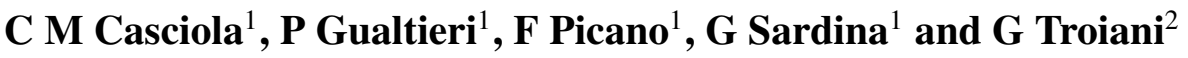 \\ ${ }^{1}$ Dipartimento di Meccanica e Aeronautica, Sapienza University, Via Eudossiana 18, 00184 Rome, Italy \\ ${ }^{2}$ ENEA C R Casaccia, Via Anguillarese 301, 00123 Rome, Italy \\ E-mail: carlomassimo.casciola@uniroma1.it
}

Received 9 November 2009

Accepted for publication 24 March 2010

Published 31 December 2010

Online at stacks.iop.org/PhysScr/T142/014001

\begin{abstract}
Turbulent mixing of small and diluted inertial particles presents many peculiar and unexpected features such as preferential segregation at small scales, i.e. clustering or, in wall flows, preferential wall accumulation, i.e. turbophoresis, which are induced by the multi-scale features of the turbulence in the carrier fluid. In the context of multi-phase flows, the effect of turbulence on particle distributions was commonly addressed in simplified geometries as in homogeneous or channel flows. The present paper discusses the dynamics of suspensions with different inertia in the far field of turbulent axisymmetric jets by means of direct numerical simulations. The jet is a well-known constant Reynolds number flow where the characteristic length scale grows linearly with distance from the jet origin, while the characteristic velocity decays in inverse proportion. These features, combined with the finite inertia, induce peculiar non-equilibrium effects on the spatial distribution of the particles. They range from spatially developing small-scale clustering, due to the multi-scale nature of the turbulent fluctuations, to self-similarity of the mean particle velocity profile, presumably collapsing on a one-parameter family of shapes parameterized in terms of the local large-scale Stokes number. The properties presented here are the most evident features of this most interesting system, where intermittency and spatial inhomogeneity interact to induce even subtler effects of spatial segregation, which certainly deserve further investigation.
\end{abstract}

PACS numbers: 47.27.wg, 47.55.Kf

(Some figures in this article are in colour only in the electronic version.)

\section{Introduction}

The mixing of a particulate phase dispersed in a turbulent jet is widely found in nature and technology. A remarkable example is the narrow stream of gas and particulate that is ejected during a Plinian volcano eruption. In this process the mixing of the entrained fresh air into the hot stream of gas is crucial in establishing the conditions for pyroclastic flow [1].

Entrainment also affects droplet evaporation/condensation in clouds [2], where the finite particle inertia enhances the collision rate [3]. The droplet-laden jet is also the prototypal flow for fuel injectors, which are ubiquitous devices found in any kind of internal combustion engine, turbine engine or rocket motor.

In a particle-laden turbulent jet, particle dynamics exhibit several features that need to be addressed in detail. Some of them are shared by other kinds of turbulent two-phase flows, such as the homogeneous isotropic flow and the homogeneous shear flow that offer simplified models to address certain generic behavior of inertial particles in turbulence. Other features are specific for the jet geometry, whose peculiar aspects, as we shall see, determine the statistical response of the particles to the turbulence.

The finite inertia of the particles leads to many non-equilibrium effects and anomalous aspects in transport phenomena. It prevents particle trajectories from taking the fluid paths a Lagrangian tracer would follow and induces small-scale clustering of the particles. Hence, the turbulent mixing never fully homogenizes a suspension of particles with finite inertia, which will be constituted mainly by multi-scale voids and clusters of particles. The issue has been thoroughly examined in isotropic (see e.g. [4-6], among others) and homogeneous shear turbulence [7, 8]. As discussed here, this phenomenology emerges also in jet flows where it becomes a local feature found at specific stations along the jet axis. 
Inhomogeneity adds new features, particularly evident in wall flows where a mean drift of particles towards the wallthe so-called turbophoresis-is apparent [9-15].

Similar effects are expected also in jets, where, however, beyond a strong inhomogeneity in the cross stream direction, the milder variation along the axis of the stream induces important new effects.

Several papers investigate the behavior of particles (e.g. [16-18]) and of evaporating droplets (e.g. $[19,20])$ in the transitional, non-universal, near-field region of jets. For heavy particles-large particle-to-fluid density ratio-particles are found to concentrate in the shear layer outside the large coherent vortical structures that populate the near field, consistent with the general trend observed in other flows. Few works $[18,21,22]$ concern the far-field behavior, where the back-reaction of particles on the fluid stream is shown to impact the spreading rate of the jet. The outward particle mean radial velocity is larger than it is for the fluid [23], suggesting localization effects associated with preferential sampling of outward fluid motions, see [15] for similar effects in the wall layer of wall turbulence. Inertial effects on particles decrease moving downstream of the jet [23], consistent with the increased time scale of the flow.

In this framework, this paper aims at analyzing the particle behavior from a direct numerical simulation (DNS) of a free jet laden with different particle populations to understand the behavior of the system in the far field.

\section{DNS of the particle-laden jet}

In order to address particle dynamics in a turbulent round jet, a Lagrangian approach to track the disperse phase is coupled to a standard Eulerian DNS approach for the fluid phase. For the very diluted suspension of tiny particles (diameter smaller than the Kolmogorov scale) considered here, inter-particle collisions and force-feedback on the fluid can be safely neglected.

The carrier fluid is assumed to obey the incompressible Navier-Stokes equations,

$$
\begin{gathered}
\nabla \cdot \mathbf{u}=0, \\
\frac{\partial \mathbf{u}}{\partial t}+\mathbf{u} \cdot \nabla \mathbf{u}=-\frac{\nabla p}{\rho}+v \nabla^{2} \mathbf{u},
\end{gathered}
$$

where $\rho$ and $v$ are the density and kinematic viscosities of the fluid, respectively, and $\mathbf{u}$ is the fluid velocity.

The inlet boundary condition for the turbulent round jet is provided by a companion DNS of a turbulent pipe flow whose instantaneous velocity field at a fixed section is used to generate the inlet data for the jet simulation. This procedure, which allows for realistic inlet fluctuations and avoids unphysical assumptions on the velocity profile, models a free jet issuing in the external environment from a fully developed pipe flow. Crucial issues for a successful DNS of free jets are the conditions enforced on the boundaries used to artificially truncate the computational domain, consisting of a side cylindrical mantle and an exit section. The traction-free boundary conditions on the side mantle and the Orlanski-type outlet condition used here were already shown to be able to provide the correct entrainment rate and to preserve the constant momentum flux of the unbounded jet [24].

The algorithm solves equation (1) in the cylindrical coordinates by a conservative second order finite difference scheme on a staggered grid, with time integration performed by an explicit third order low-storage Runge-Kutta scheme, see [24] for additional details of numerics and code validation.

The main parameter of the simulation is the bulk Reynolds number, $R e_{\mathrm{R}}=U_{\mathrm{b}} R / v=2000$, with $U_{\mathrm{b}}$ being the bulk velocity and $R$ the nozzle radius. The domain dimensions are $2 \pi \times 22 R \times 83 R$ in the azimuthal, $\theta$, radial, $r$, and axial, $z$, directions, respectively, with a corresponding non-uniform mesh of $128 \times 145 \times 784$ nodes. Overall, the grid spacing allows for a resolution nowhere worse than three Kolmogorov length scales.

The tiny, rigid, spherical particles with diameter, $d_{p}$, much smaller than the Kolmogorov scale are treated as material points with finite inertia evolving according to Newton's law. The mass density of the material forming the solid phase is taken much larger than that of the fluid, $\rho_{p} / \rho=$ 1000. In these conditions the only significant force acting on the particles is the viscous Stokes drag [4], and each particle evolves according to the equations [25]

$$
\begin{gathered}
\frac{\mathrm{d} \mathbf{v}}{\mathrm{d} t}=\frac{\mathbf{u}(\mathbf{x})-\mathbf{v}}{\tau_{\mathrm{p}}} \\
\frac{\mathrm{d} \mathbf{x}}{\mathrm{d} t}=\mathbf{v},
\end{gathered}
$$

where $\mathbf{v}$ denotes the particle velocity and $\tau_{\mathrm{p}}=\rho_{\mathrm{p}} d_{\mathrm{p}}^{2} /(\rho \nu 18)$ is the particle response time (Stokes time). The Stokes number, defined as the ratio of $\tau_{p}$ to the characteristic time scale of the carrier fluid, controls the particle dynamics for a given flow field. With the nominal flow time scale given by the ratio of nozzle radius to bulk exit velocity, the expression for the nominal Stokes number is $S t_{0}=\tau_{\mathrm{p}} U_{\mathrm{b}} / R$.

Seven populations of identical particles are considered, with Stokes number ranging between 2 and 128. For each population the injection rate is fixed at 150 particles per eddy turnover time, $R / U_{0}$. A mixed linear-quadratic formula based on Lagrange polynomials is used to interpolate the fluid velocity $\mathbf{u}$ at the particle position $\mathbf{x}$, see equation (3). The same three-stage third order low storage Runge-Kutta method used for the fluid phase evolves the particle populations. Further numerical details can be found in [15] where the same algorithm is used for the DNS simulation of a particle-laden turbulent pipe flow.

The simulation was preliminarily run up to $T=$ $1000 R / U_{0}$ to allow the carrier phase to reach a statistical steady state. Successively, particles were continuously introduced at the jet inlet. After the particle populations achieved the statistical steady state, the data acquisition procedure started collecting more than 160 time-independent fields with temporal separation of $2.5 R / U_{0}$, enough to achieve statistical convergence of the observables to be addressed in the next section.

\section{Scaling considerations}

In a free turbulent jet, the axial flux of momentum is independent of the coordinate $z$ along the axis. Sufficiently 
far from the inlet, the jet is known to achieve a self-similar state where the dimensionless axial velocity profile, $U_{z} / U_{\mathrm{c}}=$ $U_{z}^{*}(\xi)$, depends only on the reduced radial coordinate $\xi=$ $r / r_{1 / 2}(z)[24,26,27]$. Here, $U_{\mathrm{c}}(z)$ is the centerline mean velocity and $r_{1 / 2}(z)$ is the characteristic transversal length scale at $z$, typically the jet half-width defined as the radial distance from the axis where the mean axial velocity equals half its centerline value. Analogously, self-similarity is observed also for second order dimensionless statistics and dissipation [24]. Altogether, these properties imply that $\operatorname{Re}_{r_{1 / 2}}=U_{\mathrm{c}}(z) r_{1 / 2}(z) / v$ is independent of $z$. From experimental measurements, see e.g [26, 28], and numerical simulations [24, 29], it is also known that the centerline velocity in the far field decays in inverse proportion to axial distance, namely

$$
\frac{U_{\mathrm{c}}(z)}{U_{\mathrm{b}}}=\frac{B 2 R}{z-z_{0}},
$$

where $z_{0}$ is the virtual origin and $B$, a given constant for a specific jet, is expected to be a Reynolds number-independent quantity weakly dependent on the inflow details [24, 29, 30]. As a consequence, the jet half-width scales linearly with axial distance,

$$
\frac{r_{1 / 2}}{R}=S \frac{z-z_{0}}{R},
$$

with $S$ the spreading rate strictly connected to $B$ via momentum conservation $[24,26]$,

$$
R e_{r_{1 / 2}}=2 B S R e_{R}
$$

The external turbulent time scale $T_{r_{1 / 2}}$, given in terms of $r_{1 / 2}$ and $U_{\mathrm{c}}$,

$$
T_{r_{1 / 2}} \frac{U_{\mathrm{b}}}{R}=\frac{r_{1 / 2}}{U_{\mathrm{c}}} \frac{U_{\mathrm{b}}}{R}=\frac{S}{2 B} \frac{\left(z-z_{0}\right)^{2}}{R^{2}},
$$

increases as the square of the distance from the virtual origin. As a consequence, the local Stokes number, defined by comparing the particle relaxation time with $T_{r_{1 / 2}}$,

$$
S t_{r_{1 / 2}}(z)=\frac{\tau_{\mathrm{p}}}{T_{r_{1 / 2}}}=\frac{2 B}{S} \frac{R^{2}}{\left(z-z_{0}\right)^{2}} S t_{0},
$$

decays with the inverse squared distance from the origin.

Concerning the fine scales, the same similarity arguments lead to the conclusion that the typical dissipation rate in the section, e.g. its centerline value, scales as

$$
\varepsilon_{\mathrm{c}} \frac{R}{U_{\mathrm{b}}^{3}}=\hat{\varepsilon}_{\mathrm{c}} \frac{\left(U_{\mathrm{c}} / U_{\mathrm{b}}\right)^{3}}{\left(r_{1 / 2} / R\right)}=\frac{8 B^{3} \hat{\varepsilon}_{\mathrm{c}}}{S} \frac{R^{4}}{\left(z-z_{0}\right)^{4}}
$$

with $\hat{\varepsilon}_{\mathrm{c}}=\varepsilon_{\mathrm{c}} r_{1 / 2} / U_{\mathrm{c}}^{3}$ constant. The corresponding Kolmogorov length $\eta_{\mathrm{c}}=v^{3 / 4} / \varepsilon_{\mathrm{c}}^{1 / 4}$ is

$$
\frac{\eta_{\mathrm{c}}}{R}=\frac{1}{\operatorname{Re}_{R}^{3 / 4}}\left(\frac{S}{8 B^{3} \hat{\varepsilon_{\mathrm{c}}}}\right)^{1 / 4} \frac{z-z_{0}}{R}
$$

with the dissipative time scale, $t_{\eta}=\eta^{2} / \nu$, given by

$$
\tau_{\eta} \frac{U_{\mathrm{b}}}{R}=\frac{1}{\operatorname{Re}_{R}^{1 / 2}}\left(\frac{S}{8 B^{3} \hat{\varepsilon_{\mathrm{c}}}}\right)^{1 / 2} \frac{\left(z-z_{0}\right)^{2}}{R^{2}} .
$$

In these conditions the ratios between large- and small-scale quantities present the usual dependence on Reynolds number,

$$
\begin{gathered}
\frac{r_{1 / 2}}{\eta_{\mathrm{c}}}=\operatorname{Re}_{R}^{3 / 4}\left((2 S B)^{3} \hat{\varepsilon}_{\mathrm{c}}\right)^{1 / 4}=\operatorname{Re}_{r_{1 / 2}}^{3 / 4} \hat{\varepsilon}_{\mathrm{c}}^{1 / 4}, \\
\frac{T_{r_{1 / 2}}}{t_{\eta}}=\operatorname{Re}_{R}^{1 / 2}\left(2 S B \hat{\varepsilon}_{\mathrm{c}}\right)^{1 / 2}=\operatorname{Re}_{r_{1 / 2}}^{1 / 2} \hat{\varepsilon}_{\mathrm{c}}^{1 / 2},
\end{gathered}
$$

and are independent of the axial coordinate.

In a jet, relations (13) and (14) are the main tools to estimate the impact of the inertia on particle dynamics. Actually, after defining a small-scale Stokes number as the ratio of $\tau_{p}$ to the Kolmogorov time $t_{\eta}$,

$$
S t_{\eta}=\frac{\tau_{p}}{t_{\eta}}=S t_{0} \operatorname{Re}_{R}^{1 / 2}\left(\frac{8 B^{3} \hat{\varepsilon}_{\mathrm{c}}}{S}\right)^{1 / 2} \frac{R^{2}}{\left(z-z_{0}\right)^{2}},
$$

the effect of the inertia is found to decrease quadratically with the distance from the origin. This implies that all particles eventually behave as tracers sufficiently downstream of the exit section of the jet. The ratio between $S t_{\eta}$ and $S t_{r_{1 / 2}}$ is constant along the axis of the jet and is determined by the Reynolds number,

$$
\frac{S t_{\eta}}{S t_{r_{1 / 2}}}=R e_{r_{1 / 2}}^{1 / 2} \hat{\varepsilon}_{\mathrm{c}}^{1 / 2} \propto R e_{R}^{1 / 2}
$$

The behavior of a given particle changes continuously along the axis of the jet, from strongly inertia dominated near the jet exit to purely Lagrangian far downstream. An intermediate region exists where an inertial particle may behave as a 'tracer' with respect to the large-scale motions $S t_{r_{1 / 2}}(z) \ll 1$, still presenting substantial inertial effects with respect to the small-scale velocity fluctuations $S t_{\eta}(z)>1$. The extension of this region, $z_{2}^{i}-z_{1}^{i}$, is determined by the conditions

$$
\begin{aligned}
& S t_{r_{1 / 2}}\left(z_{1}^{i}\right)=S t_{0}\left(2 B R^{2} / S\right) /\left(z_{1}^{i}-z_{0}\right)^{2}=a, \\
& S t_{\eta}\left(z_{2}^{i}\right)=S t_{0}\left(8 R^{4} B^{3} \hat{\varepsilon}_{\mathrm{c}}^{1 / 2} / S\right)^{1 / 2} R e_{R}^{1 / 2} /\left(z_{2}^{i}-z_{0}\right)^{2}=b
\end{aligned}
$$

with $a \simeq b \ll 1$. Hence, the larger the inertia, the farther downstream the intermediate region is shifted, $z_{1}^{i}-z_{0} \propto$ $\sqrt{S t_{0}}$. The extension of the intermediate region depends on the particle relaxation time and scales with Reynolds number to the power one-fourth $z_{2}^{i}-z_{1}^{i} \propto R e_{R}^{1 / 4} \sqrt{S t_{0}}$, $R e_{R} \gg 1$. For $z_{1}^{i}<z<z_{2}^{i}$ we should expect small-scale clustering, controlled by the dissipative Stokes number $S t_{\eta}$, together with a passive-tracer-like dynamics of large-scale and single point statistics, such as mean particle velocity or fluctuation intensity, which are controlled by $S t_{r_{1 / 2}}$.

The dimensional reasoning illustrated above allows for the qualitative prediction of the kind of motion executed by a heavy particle while transported downstream. The particle will start with purely ballistic behavior at the exit of the jet that will continue until a first critical section, $z_{\mathrm{c}}$, is reached. Here its large-scale Stokes number falls below a threshold and the particle begins to perceive the large-scale fluctuations of the turbulence. Moving downstream, the range of scales able to couple with the particle dynamics grows towards 

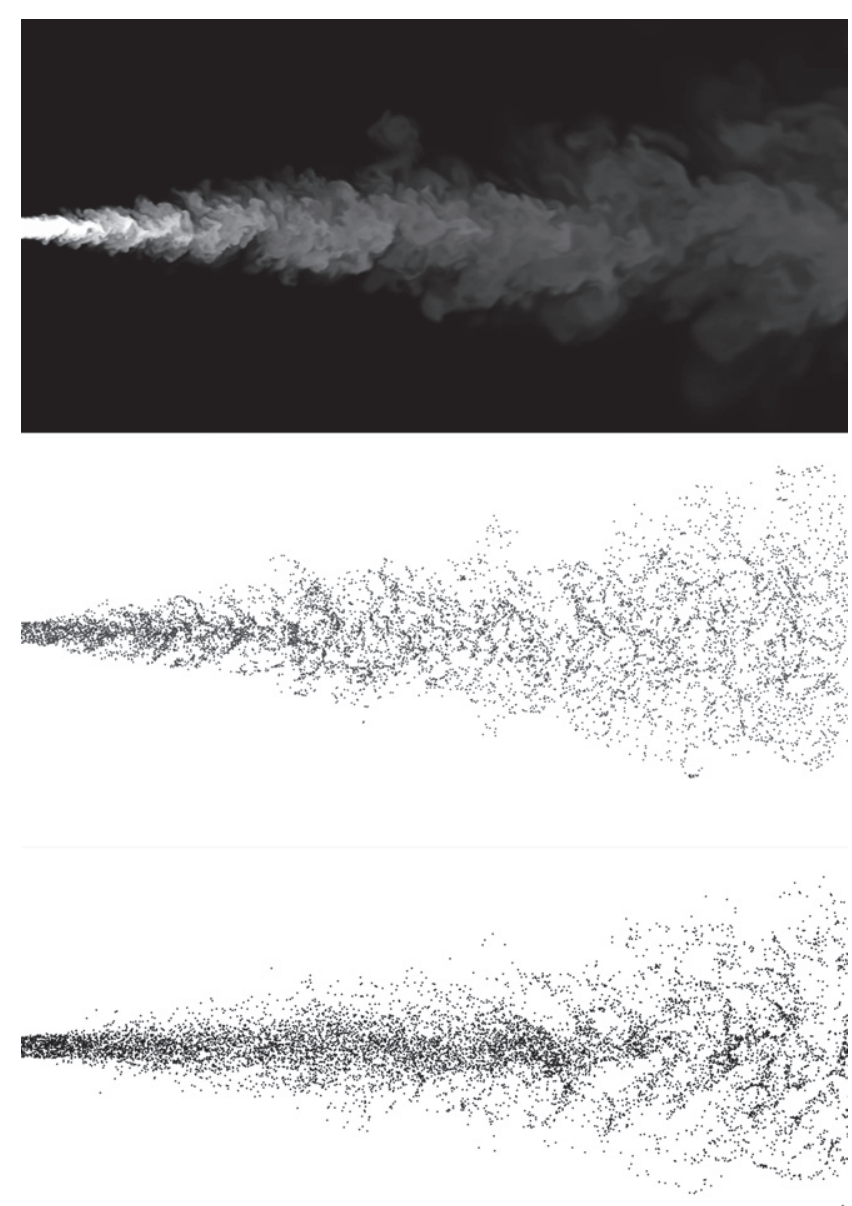

Figure 1. Instantaneous configuration of a thin axial-radial slice of the turbulent jet. Top panel: passive scalar field (Schmidt number 0.7 ). Middle panel: particles with $S t_{0}=2$. Bottom panel: particles with $S t_{0}=16$.

the small scales. At station $z_{1}^{i}$ the particle attains tracer-like behavior with respect to large-scale fluctuations, until after passing through section $z_{2}^{i}$ the particle eventually becomes a Lagrangian tracer in every respect. At any given station beyond $z_{\mathrm{c}}$ and before $z_{2}^{i}$, a range of turbulent scales exists able to induce multi-scale features in the particle population, along the lines described for homogeneous shear turbulence in [8].

\section{Results}

A visual impression of the turbulent jet is provided in the top panel of figure 1, which displays the instantaneous configuration of a transported passive scalar with Schmidt number 0.7 . Given fully turbulent inflow, no evidence is found of the Kelvin-Helmholtz instability that classically characterizes the near field of jets with a laminar (top-hat) inflow. Beyond 20-25 $R$ from the origin, the jet shows the expected self-similar behavior for mean velocities and Reynolds stresses, see [24] and section 3. Also the scalar field conforms to the expected behavior, see e.g. [31].

The other two panels of figure 1 show the instantaneous configuration of two different particle populations, $S t_{0}=2$ and 16 for the middle and bottom panels, respectively. The

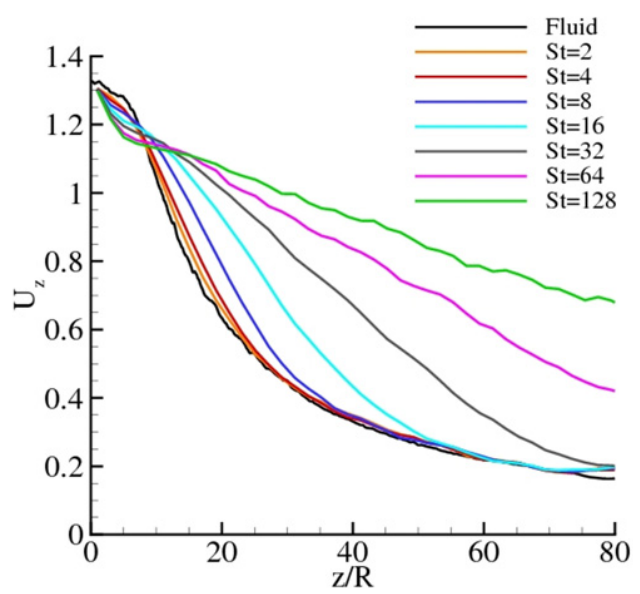

Figure 2. Centerline mean velocity versus $z / R$ for several particle populations. The black line provided for reference is the fluid velocity.

region near the jet nozzle is dominated by inertia for both populations, with the heaviest particles moving straight along the axis for a length of about $5 R$ without perceiving fluid fluctuations. Close to the axis the population at $S t_{0}=16$ keeps an almost uniform distribution up to a significantly long distance $(\sim 50 R)$. In this region these particles execute purely ballistic motions. In contrast, in the same region, both the passive scalar field and the lightest particles show large fluctuations in the local concentration.

Concerning the lightest particles, the occurrence of small-scale clustering is apparent in the central region of the image. This impression is substantiated by evaluating the local small-scale Stokes number $S t_{\eta}$, which lies in the range $0.5-2.2$ for $30 \leqslant z / R \leqslant 60$ (here $B=6.77, z_{0} / R=-0.8$, $S=0.089$ and $\hat{\epsilon}_{\mathrm{c}}=0.018$, as obtained from the analysis of centerline mean velocity, spreading rate and dissipation [24]). This behavior is consistent with the results found in a homogeneous turbulent shear flow and in isotropic turbulence where peak clustering occurs where $S t_{\eta} \simeq 1$ [4, 8]. The overall picture provided by the scaling analysis of section 3 is confirmed by the behavior of the particles with $S t_{0}=16$, which begin to manifest significant clustering effects towards the right edge of the image where the local small-scale Stokes number becomes sufficiently small, $S t_{\eta} \sim 2.2$. Hence, the data confirm the idea that the small-scale particle distribution (small-scale mixing) is ruled by the local $S t_{\eta}$.

As far as the far field is concerned, the mean centerline velocity of particles with $S t_{0}=8$ almost matches the fluid centerline mean velocity $U_{\mathrm{c}}$ at $z \simeq 40 R$, see figure 2 where data for several populations are reported. At station $z=40 R$, equation (9) yields $S t_{r_{1 / 2}} \simeq 0.73$. The same value of the local large-scale Stokes number $S t_{r_{1 / 2}}$ is achieved at $z \simeq 57 R$ for particles with $S t_{0}=16$. Exactly at this station these kinds of particles match the fluid mean centerline velocity (figure 2). On the basis of the scaling analysis of section 3 , particles with $S t_{0}=32$ should match the fluid average velocity at $z \simeq 81 R$, close to the artificial boundary of the computational domain. Figure 2 seems to confirm this trend, although a certain bias is certainly induced by the numerical outlet boundary condition that may locally alter the correct jet dynamics.

By conforming to the predictions of the scaling theory provided in section 3 , these results suggest that the centerline 

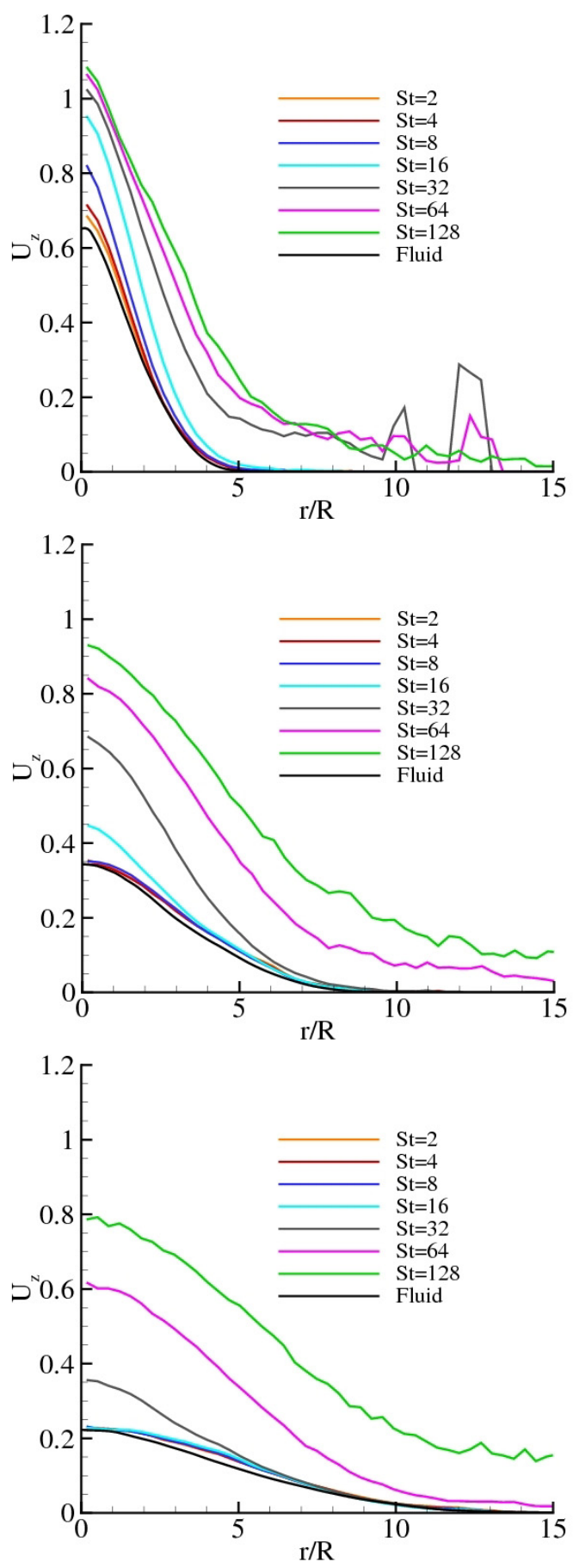
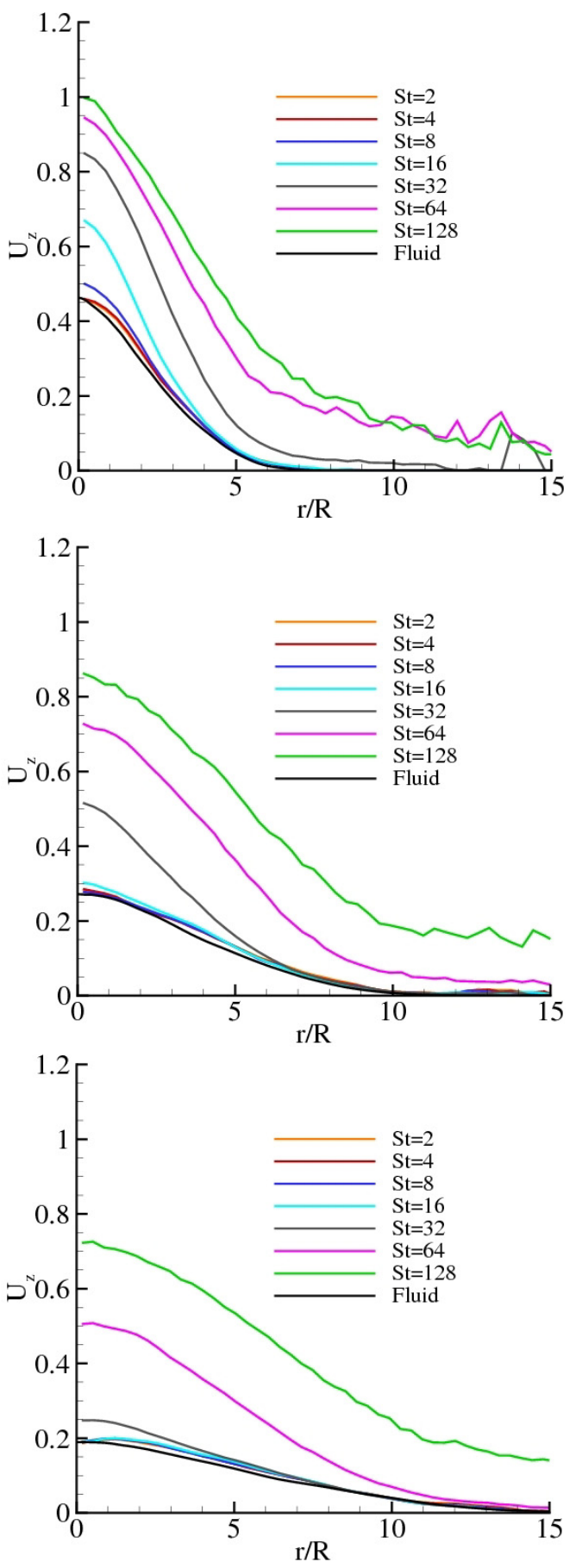

Figure 3. Mean axial particle velocity profiles versus $r / R$ for several particle populations at different axial stations. In each panel the fluid profile is plotted in black. Top panels: left, $z / R=20$; right, $z / R=30$. Middle panels: left, $z / R=40$; right, $z / R=50$. Bottom panels: left, $z / R=60 ;$ right, $z / R=70$.

mean velocity of the solid phase, $V_{\mathrm{c}}$, is controlled solely by the local large-scale Stokes number $S t_{r_{1 / 2}}$. In other words, after normalizing with the local fluid mean centerline velocity, the dimensionless velocity $V_{\mathrm{c}} / U_{\mathrm{c}}$ of particles with different $\tau_{p}$ should be the same at stations where $S t_{r_{1 / 2}}(z)$ is the same. This notion is straightforwardly extended to the conjecture that the whole mean axial velocity profile across the jet section could be self-similar if appropriately rescaled and parameterized with the local large-scale Stokes number.

The radial profiles of the mean axial velocity for fluid and particle populations are shown in figure 3 at several axial distances. At fixed station, with increasing $\tau_{p}$, particle velocities are found to exceed the fluid velocity and to present a wider distribution across the section. These are in fact memory effects, with particles retaining memory of their previous exposure to fluid velocities, which are on average faster, since the fluid velocity decreases along the average streamlines. For a specific particle population, moving downstream a station is eventually reached where the particle velocity profile exactly reproduces the fluid, see e.g. at $z=30 R$ particles with $S t_{0} \leqslant 4$. Looking at the data, the particles begin to recover the fluid velocity starting from the outer part of the jet, eventually achieving the coincidence of velocity on the axis further downstream, e.g. compare the 


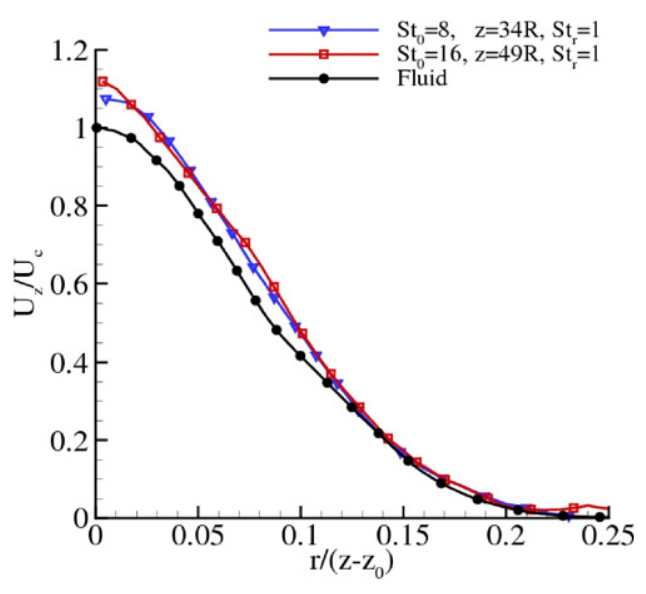

Figure 4. Normalized mean axial particle velocity $V / U_{\mathrm{c}}(z)$ as a function of the reduced radial coordinate $r /\left(z-z_{0}\right)$ for two particle populations ( $S t_{0}=8$ and 16 , triangles and squares, respectively) at two corresponding stations $(z=34 R$ and $49 R$, for the former and the latter population, respectively) such that the local large-scale Stokes number achieves in both cases unit value $\left(S t_{r_{1 / 2}}=1\right)$. The filled circles provide the mean fluid axial velocity.

profiles of particles with $S t_{0}=16$ at $z=40 R$ and at $z=50 R$. A similar trend is found for $S t_{0}=32$, where things are shifted downstream. In this case the end of the computational domain is reached before particles can completely acquire the fluid average velocity.

Since the matching between particle and fluid mean velocities starts from the outer part of the jet, once particles attain the fluid velocity at the axis of the jet the entire profile collapses on the fluid profile. In our simulations this is found to occur within reasonable accuracy from the station where $S t_{r_{1 / 2}}(z) \leqslant 0.73$ onwards. From equation (9) we estimate that the mean particles velocity profile reproduces the fluid beyond the station $z_{\mathrm{f}}$,

$$
z>z_{\mathrm{f}}=z_{0}+\sqrt{\frac{2 B}{0.73 S} S t_{0}} R \simeq 13.5 \sqrt{S t_{0}} R,
$$

where the coordinate $z_{0}$ of the virtual origin of the jet is typically of the order of $R$ and negative.

The conjecture that the entire mean particle velocity profile is self-similar at corresponding axial stations is checked in figure 4 . The figure compares the mean particle velocity profile normalized by the local mean centerline fluid velocity $U_{\mathrm{c}}(z)$ for two different populations. The normalized profile is plotted as a function of the reduced radial coordinate $r /\left(z-z_{0}\right)$, which is the similarity variable for the mean fluid velocity profile, see section 3 . For each population the profile is taken at the station where the corresponding large-scale Stokes number $S t_{r_{1 / 2}}(z)$ attains unit value. The shape similarity of the two profiles is striking.

Other features emerge clearly from the plots in figure 4. The selected unit value of the local Stokes number exceeds 0.73 , which is the limit below which particle and fluid profiles become indistinguishable. The particle average velocity is already very close to that of the fluid, almost everywhere in the section. However, while the external portion of the profile is already exactly on top of the fluid one, the inner portion still shows a significant discrepancy, exceeding the fluid velocity by $10 \%$ at the centerline. This seems to confirm to a large extent the self-similar nature of the particle average profile in the far field of the jet. A wider analysis is at present under way to complete the illustration of the conjectured self-similarity, and to look in more detail at subtler effects induced by the intermittency of the field. The results will be briefly reported elsewhere.

\section{Final remarks}

The dynamics of inertial particles dispersed in a turbulent jet has been investigated numerically by means of a DNS. This is the only DNS of the far field of a particle-laden free turbulent jet we are aware of. It required a precise validation of the carrier fluid dynamics, easily spoiled by numerical artifacts induced by the artificial boundary conditions needed to truncate the otherwise unbounded computational domain. The successful simulation of a fully developed free turbulent jet makes possible the comparison with the classical similarity theory of the jet far field. This theory was exploited here to predict the most significant features of inertial particle transport. Overall, a remarkable correspondence is found between the proposed theoretical predictions and the results of our numerical experiments.

From the theoretical point view, two different Stokes numbers basically rule the dynamics of the particles. One, $S t_{\eta}=\tau_{\mathrm{p}} / \tau_{\eta}$, compares the particle relaxation time with the local Kolmogorov time scale. The other, $S t_{r_{1 / 2}}=\tau_{\mathrm{p}} / T_{r_{1 / 2}}$, accounts for the local large-scale eddy-turnover time of the jet, $T_{r_{1 / 2}}=r_{1 / 2} / U_{\mathrm{c}}$. The former controls the small-scale particle dynamics that, as established in statistically homogeneous flows, is prone to small-scale clustering in the appropriate Stokes number range. The latter describes the response to large-scale fluctuations and controls low-order single point statistics.

For a fixed particle inertia, both these parameters are found to decay as the square of the distance from the origin of the jet. As a consequence, independently of the specific inertia, any particle is bound to eventually behave as a Lagrangian tracer at sufficient distance from the jet orifice. The ratio between the small- and the large-scale Stokes number is found to be proportional to the square root of the jet Reynolds number $R e_{R}$, thereby providing predictions on the range of scales influenced by the finite particle inertia and the spatial extension of the region where clustering effects take place. Consistently, in a certain intermediate range of positions along the jet axis, a given particle behaves as if it were a Lagrangian tracer with respect to large-scale fluid velocity fluctuations, while still obeying the ballistic limit with respect to the small-scale dynamics.

On the basis of theoretical considerations, the mean axial particle velocity profile is conjectured to achieve a self-similar state in the far field of the jet, where the scaling parameter is the local large-scale Stokes number, based on the flow time scale of the local section of the jet. The conjecture is verified by the numerical data to very good accuracy.

As a final comment, we like to stress that those discussed here are only the gross properties of this extremely interesting system. Small-scale intermittency of fluid fluctuations in conjunction with the spatial inhomogeneity of the field induces subtler effects, which are at present under 
investigation and will be reported in detail elsewhere. In addition, we consider it worth mentioning here that especially interesting features are expected to emerge at the boundary of the jet, where the so-called external intermittency is expected to play a major role.

\section{References}

[1] Kaminski E, Tait S and Carazzo G 2005 Turbulent entrainment in jets with arbitrary buoyancy J. Fluid Mech. $526361-76$

[2] Kostinski A B and Shaw R A 2001 Scale-dependent droplet clustering in turbulent clouds J. Fluid Mech. 434 389-98

[3] Falkovich G, Fouxon A and Stepanov M G 2002 Acceleration of rain initiation by cloud turbulence Nature 419 151-4

[4] Bec J, Biferale L, Cencini M, Lanotte A, Musacchio S and Toschi F 2007 Heavy particle concentration in turbulence at dissipative and inertial scales Phys. Rev. Lett. 98084502

[5] Goto S and Vassilicos J C 2008 Sweep-stick mechanism of heavy particle clustering in fluid turbulence Phys. Rev. Lett. 10054503

[6] Reade W C and Collins L R 2000 Effect of preferential concentration on turbulent collision rates Phys. Fluids 122530

[7] Shotorban B and Balachandar S 2006 Particle concentration in homogeneous shear turbulence simulated via Lagrangian and equilibrium Eulerian approaches Phys. Fluids 18065105

[8] Gualtieri P, Picano F and Casciola C M 2009 Anisotropic clustering of inertial particles in homogeneous shear flow J. Fluid Mech. 629 25-39

[9] Caporaloni M, Tampieri F, Trombetti F and Vittori O 1975 Transfer of particles in nonisotropic air turbulence J. Atmos. Sci. 32 565-8

[10] Reeks M W 1983 The transport of discrete particles in inhomogeneous turbulence J. Aerosol Sci. 14 729-39

[11] Young J and Leeming A 1997 A theory of particle deposition in turbulent pipe flow J. Fluid Mech. 340 129-59

[12] Kaftori D, Hetsroni G and Banerjee S 1995 Particle behavior in the turbulent boundary layer. I. Motion, deposition and entrainment Phys. Fluids 7 1095-106

[13] Rouson D W I and Eaton J K 2001 On the preferential concentration of solid particles in turbulent channel flow J. Fluid Mech. 428 149-69

[14] Picciotto M, Marchioli C and Soldati A 2005 Characterization of near-wall accumulation regions for inertial particles in turbulent boundary layers Phys. Fluids 17098101
[15] Picano F, Sardina G and Casciola C M 2009 Spatial development of particle-laden turbulent pipe flow Phys. Fluids 21093305

[16] Sbrizzai F, Verzicco R, Pidria M F and Soldati A 2004 Mechanisms for selective radial dispersion of microparticles in the transitional region of a confined turbulent round jet Int. J. Multiph. Flow 30 1389-417

[17] Fan J, Luo K, Ha M Y and Cen K 2004 Direct numerical simulation of a near-field particle-laden plane turbulent jet Phys. Rev. E 7026303

[18] Longmire E and Eaton J 1992 Structure of a particle-laden round jet J. Fluid Mech. 236217

[19] Almeida T G and Jaberi F A 2006 Direct numerical simulations of a planar jet laden with evaporating droplets Int. J. Heat Mass Transfer 49 2113-23

[20] Selle L C and Bellan J 2007 Characteristics of transitional multicomponent gaseous and drop-laden mixing layers from direct numerical simulation: composition effects Phys. Fluids 19063301

[21] Foreman R J and Nathan G J 2009 Scaling of the gas phase in particle-laden turbulent axisymmetric jets Int. J. Multiph. Flow 35 96-100

[22] Hardalupas Y, Taylor A M K P and Whitelaw J H 1989 Velocity and particle-flux characteristic of turbulent particle-laden jets Proc. R. Soc. A 426 31-78

[23] Prevost F, Boree J, Nuglisch H J and Charnay G 1996 Measurements of fluid/particle correlated motion in the far field of an axisymmetric jet Int. J. Multiph. Flow 22 685-701

[24] Picano F and Casciola C M 2007 Small-scale isotropy and universality of axisymmetric jets Phys. Fluids 19118106

[25] Maxey M R and Riley J J 1983 Equation of motion for a small rigid sphere in a nonuniform flow Phys. Fluids $\mathbf{2 6} 883$

[26] Hussein H J, Capp S P and George W K 1994 Velocity measurements in a high-Reynolds-number momentum-conserving, axisymmetric turbulent jet $J$. Fluid Mech. 25831

[27] Pope S B 2008 Turbulent Flows (Cambridge: Cambridge University Press)

[28] Panchapakesan N R and Lumley J L 1993 Turbulence measurements in axisymmetric jets of air and helium. Part 1. air jet J. Fluid Mech. 246197

[29] Boersma B J, Brethouwer G and Nieuwstadt F T M 1998 A numerical investigation on the effect of inflow conditions on the self-similar region of a round jet Phys. Fluids 10899

[30] George W K 1989 Self-preservation of turbulent flows and its relation to initial conditions and turbulent structures Advances in Turbulence (New York: Hemisphere) p 39

[31] Mi J, Nobes D S and Nathan G J 2001 Influence of jet exit conditions on the passive scalar field of an axisymmetric free jet J. Fluid Mech. 432 91-125 